\title{
The evolution of Bernstein modes in quantum wires with increasing deviation from parabolic confinement
}

\author{
Arne Brataas† Vidar Gudmundsson $\ddagger$ A G Mal'shukov§ and K A Chao† \\ $\dagger$ Department of Physics, Norwegian University of Science and Technology, N-7034 \\ Trondheim, Norway. \\ $\ddagger$ Science Institute, University of Iceland, Dunhaga 3, IS-107 Reykjavik, Iceland. \\ $\S$ Institute of Spectroscopy, Russian Academy of Sciences, 142092 Troitsk, Moscow \\ Region, Russia
}

\begin{abstract}
We investigate the evolution of the interaction of the magnetoplasmon resonance with the harmonics of the cyclotron resonance as the confinement of an electron gas in a quantum wire increasingly deviates from the parabolic case. The occurrence of the Bernstein modes is observed in a time-dependent Hartree model of a two-dimensional electron gas in a single quantum wire.
\end{abstract}

\section{Introduction}

Quantum wires are interesting for probing the electron-electron interaction in the transition regime from quasi two-dimensional systems to quasi one-dimensional systems. These systems can be fabricated starting from two dimensional semiconductor microstructures (2DES), for example in AlGaAs-GaAs heterostructures. The wires may be created by a gate structure or by etching techniques. The presence of a magnetic field is manifest by many interesting quantum features, most notably the quantum Hall effect and the fractional quantum Hall effect. Far infrared response is a powerful method for determining the charge-density spectra in the transverse direction. For transverse parabolic potentials, the motion of the electrons may be separated into center-of-mass motion and relative motion. The center-of-mass motion is decoupled from the relative motion of the electrons, and takes place in a transverse potential with the effective frequency $\left(\omega_{0}^{2}+\omega_{c}^{2}\right)^{1 / 2}$, where $\hbar \omega_{0}$ is the confinement energy and $\omega_{c}$ is the cyclotron frequency. The time-dependent external potential $\phi_{e}$ representing the long wave length far-infrared radiation only couples to the center-of-mass motion causing a single peak in the FIR spectrum at the plasmon frequency $\Omega_{p}=\left(\omega_{c}^{2}+\omega_{0}^{2}\right)^{1 / 2}$ equal to the effective transverse frequency [1], 2]. Therefore, according to Kohns theorem[四], although a rich 
spectrum would be expected, there is only a single resonance. For small systems with general confinement potentials deviating strongly from the parabolic case, the singleparticle excitations may overlap with the collective excitations causing a fine-structure in the plasmon peak [3, 4. Any deviation from parabolic confinement will couple the internal and collective motion.

Bernstein showed[5] that in 3D homogeneous systems the dispersion of the magnetoplasmon as a function of the magnetic field will have anticrossings at finite wave vectors $(\vec{k} \perp \vec{B})$ around $\Omega_{p}=n \omega_{c}$ where $n=2,3, \cdots$. The calculation was based on solving the classical Boltzmann equation and the Maxwell's equations self-consistently. The strength of the anticrossings depends on temperature, wave length and magnetic field in a complex way and decreases for the higher harmonics. An interaction between the magnetoplasmon resonance and the harmonics of the cyclotron resonance, a so-called Bernstein mode [5], have been observed in 3DES[6], 2DES[7, 8], 1DES (wires) and 0DES (dots) 田. For confined electron systems in reduced dimensions the breakdown of Kohns theorem in nonparabolic potentials is sufficient to observe the Bernstein modes instead of considering absorption at a finite wave vector.

We study the far infrared absorption in the integer quantum Hall regime. Here we consider a broad range of deviations from parabolic wires in the time-dependent Hartree approximation. Increasing deviations give richer excitation spectra when the magnetoplasmon resonance starts to couple to increasingly higher harmonics of the cyclotron resonance. We systematically investigate how the splitting and its location depend on the strength of the deviation from the parabolic confinement.

\section{Model}

We consider a strictly two-dimensional electron system lying in the $x-y$ plane. The motion in the $z$ direction is neglected since the electrons are confined to the lowest subband at the low temperature attained in experiments. We use the Hartree approximation (HA) to reduce the many-particle Hamiltonian to a single-particle Hamiltonian for each electron in an effective potential approximating the electronelectron interaction. For the FIR absorption we use the corresponding time-dependent approximation describing the self-consistent linear response of the 2DES to an external homogeneous time-varying electrical field (the random phase approximation, RPA). In a constant perpendicular magnetic field $B$ the cyclotron frequency and the magnetic length are $\omega_{c}=e B /\left(m^{*} c\right)$ and $l_{c}=\left[\hbar /\left(m^{*} \omega_{c}\right)\right]^{1 / 2}$, respectively, where $m^{*}$ is the effective mass. The dielectric constant of the surrounding medium is noted by $\kappa$. It is convenient to introduce the constant perpendicular magnetic field with the vector potential in the Landau gauge $\vec{A}(\vec{r})=(-B y, 0)$.

The effective Hamilton operator for a single electron in the confining potential $V_{c}(y)$ 
is

$$
H=-\frac{\hbar^{2}}{2 m^{*}}\left(\nabla^{2}-\frac{2 i}{l_{c}^{2}} y \frac{\partial}{\partial x}\right)+\frac{1}{2} m^{*} \omega_{c}^{2} y^{2}+V_{c}(y)+V_{H}(\vec{r}),
$$

where $V_{H}(\vec{r})$ is the self-consistent Hartree potential representing the direct Coulomb interaction between one electron and the total charge density of the 2DES. Periodic boundary condition in the longitudinal direction of the wire gives a Bloch-type singleparticle wave function

$$
\Psi_{n k}(x, y)=\frac{1}{\sqrt{L_{x}}} e^{i k x} \psi_{n k}(y),
$$

where the longitudinal wave vector $k=p \cdot 2 \pi / L_{x}$ with $p \in Z$ and $n$ is the transverse quantum number. The length of the wire, $L_{x}$, is assumed to be much larger than the effective width of the wire, therefore the Hartree potential only depends on the transverse coordinate[9]

$$
V_{H}(y)=-\frac{2 e^{2}}{\kappa} \int_{-\infty}^{\infty} d y^{\prime} n_{s}\left(y^{\prime}\right) \ln \left|\frac{y-y^{\prime}}{L}\right| .
$$

with $L$ given below. It has been assumed that a neutralising background charge exists. For noninteracting electrons in a simple one-dimensional parabolic potential $V(y)=\frac{1}{2} m^{*} \omega_{0}^{2} y^{2}$, the single-particle eigenfunctions are

$$
\phi_{n k}(y)=\frac{1}{\sqrt{L}} \frac{1}{\sqrt{2^{n} n ! \sqrt{\pi}}} H_{n}\left(\frac{y-y_{k}}{L}\right) \exp \left[-\frac{\left(y-y_{k}\right)^{2}}{2 L^{2}}\right],
$$

where $y_{k}=k \cdot l_{c}^{2} l_{0}^{4} /\left(l_{0}^{4}+l_{c}^{4}\right)$ is the center coordinate, which generally does not equal the expectation value of the transverse coordinate $y[10]$. The $n$th Hermite polynomial is denoted by $H_{n}$. The confinement length is defined as $l_{0}=\left[\hbar /\left(m^{*} \omega_{0}\right)\right]^{1 / 2}$. The electron is localised within the effective length $L=\left[\hbar /\left(m^{*} \Omega\right)\right]^{1 / 2}$, replacing the magnetic length $l_{c}$, and the cyclotron frequency $\omega_{c}$ is replaced by an effective frequency defined by $\Omega=\left(\omega_{0}^{2}+\omega_{c}^{2}\right)^{1 / 2}$. The eigenenergies corresponding to the eigenstates (四) are $E_{n k}=\hbar \Omega(n+1 / 2)+\left(\hbar^{2} k^{2} / 2 m^{*}\right) \times l_{c}^{4} /\left(l_{c}^{4}+l_{0}^{4}\right)$. In the case of vanishing confinement, (2DES) $l_{0} \rightarrow \infty$, the energy bands reduce to the familiar dispersionless Landau levels. The effective single-particle Hamiltonian is diagonalised using the wave functions of the noninteracting electrons (田) as a functional basis and the self-consistent solutions are obtained by iteration.

The response to a time-dependent perturbation may be found by the random phase approximation where exchange and correlation effects are neglected. In this meanfield approximation the noninteracting Hartree quasi-particles move in a self-consistent potential given by the external perturbation and the response of the charge density of the electrons $n_{s}$. The selfconsistent potential, $\phi_{s}(\vec{r}, \omega)$, may be found from the external 
potential $\phi_{e}(\vec{r}, \omega)$

$$
\left\langle\alpha\left|\phi_{s}(\omega)\right| \beta\right\rangle=\left\langle\alpha\left|\phi_{e}(\omega)\right| \beta\right\rangle+\sum_{\delta \gamma} \frac{H_{\alpha, \beta ; \gamma, \delta}\left(f_{\gamma}-f_{\delta}\right)}{\hbar \omega+\left(\epsilon_{\gamma}-\epsilon_{\delta}\right)+i 0^{+}}\left\langle\delta\left|\phi_{s}(\omega)\right| \gamma\right\rangle,
$$

where $\alpha$ denotes the longitudinal and transverse quantum numbers $n$ and $k . f_{\alpha}$ is the Fermi occupation factor and the Hartree matrix elements are defined in terms of the Hartree ground state wave functions

$$
H_{\alpha, \beta ; \gamma, \delta}=\frac{e^{2}}{\kappa} \int d \vec{r} \int d \vec{r}^{\prime} \frac{\psi_{\gamma}^{*}\left(\vec{r}^{\prime}\right) \psi_{\delta}\left(\vec{r}^{\prime}\right) \psi_{\alpha}^{*}(\vec{r}) \psi_{\beta}(\vec{r})}{\left|\vec{r}-\vec{r}^{\prime}\right|} .
$$

A method to find $\phi_{s}$ based on a Fourier transformation of the position coordinates and repeatedly solving (5) for all the needed values of $\omega$ is shown in Ref. [4]. Here we present an alternative method transforming (5) such that $\phi_{s}(\omega)$ can be simultaneously obtained for all values of $\omega$ from a matrix eigenvalue problem. The latter method considerably speeds up numerical calculations and we have checked that they deliver exactly the same results. The equation for the selfconsistent potential (5) simplifies for transverse external fields. The longitudinal quantum number $k$ is conserved in the transition, so that each single electron transition may be labeled by $k$ and two transverse quantum numbers $n$ and $m$. By applying a time dependent but spatially constant electric field, $\vec{E}_{e}(\vec{r}, t)=-\hat{y} \mathcal{E}_{e} \exp (-i \omega t)$, the Fourier component of the external potential is $\phi_{e}(\vec{r}, \omega)=-e \mathcal{E}_{e} y$, where $-e$ is the electron charge. In the basis (2) the interband matrix elements of the external potential $\left\{\phi_{e}(\omega)\right\}_{n, m}^{k}=\int d y \psi_{n k}(y) \phi_{e}(\vec{r}, \omega) \psi_{m k}(y)$ are real and symmetric. Since the Hartree potential is local the matrix elements of the selfconsistent potential are also symmetric with respect to the transverse quantum numbers $\left\{\phi_{s}(\omega)\right\}_{n, m}^{k}=\left\{\phi_{s}(\omega)\right\}_{m, n}^{k}$. This is also the case for local-density approximations, but not for the Hartree-Fock approximation, since the Fock potential is nonlocal[11]. The equation for the self-consistent potential may then be written as an eigenvalue problem

$$
\begin{gathered}
\left(\hbar^{2} \omega^{2}-\left(\epsilon_{n, m}^{k}\right)^{2}\right)\{\eta(\omega)\}_{n, m}^{k}=\sqrt{2 f_{n, m}^{k} \epsilon_{n, m}^{k}}\left\{\phi_{e}(\omega)\right\}_{n, m}^{k} \\
-2 \sum_{n^{\prime}, m^{\prime}, k^{\prime}}^{\epsilon_{n^{\prime}, m^{\prime}}^{k^{\prime}}>0} H_{n, m ; n^{\prime}, m^{\prime}}^{k ; k^{\prime}} \sqrt{f_{n, m}^{k} \epsilon_{n, m}^{k}} \sqrt{f_{n^{\prime}, m^{\prime}}^{k} \epsilon_{n^{\prime}, m^{\prime}}^{k^{\prime}}}\{\eta(\omega)\}_{n^{\prime}, m^{\prime}}^{k^{\prime}},
\end{gathered}
$$

where $\epsilon_{n, m}^{k}=\epsilon_{n}^{k}-\epsilon_{m}^{k}$ are the quasi-particle excitation energies and $f_{n, m}^{k}=f_{m}^{k}-f_{n}^{k}$ are the differences in occupation between initial and final states. For convenience we have introduced the variable $\{\eta(\omega)\}_{n, m}^{k}=\left\{\phi_{s}(\omega)\right\}_{n, m}^{k}\left(2 f_{n, m}^{k} \epsilon_{n, m}^{k}\right)^{1 / 2} /\left(\hbar^{2} \omega^{2}-\left(\epsilon_{n, m}^{k}\right)^{2}\right)$. The resonances in the FIR spectrum are the eigenvalues of the symmetric matrix

$$
A_{n, m ; n^{\prime}, m^{\prime}}^{k ; k^{\prime}}=\left(\epsilon_{n, m}^{k}\right)^{2} \delta_{n, m ; n^{\prime}, m^{\prime}}^{k ; k^{\prime}}-2 H_{n, m ; n^{\prime}, m^{\prime}}^{k ; k^{\prime}}\left(f_{n, m}^{k} \epsilon_{n, m}^{k} f_{n^{\prime}, m^{\prime}}^{k^{\prime}} \epsilon_{n^{\prime}, m^{\prime}}^{k^{\prime}}\right)^{1 / 2} .
$$

The power absorption may be found from the Joule heating

$$
P(\omega)=\frac{1}{2} \int d \vec{r} \operatorname{Re}\left\{\delta \vec{J}(\vec{r}, \omega) \vec{E}_{s}^{*}(\vec{r}, \omega)\right\},
$$


where $\delta \vec{J}(\vec{r}, \omega)$ is the induced current in the wire and $\vec{E}_{s}$ is the self-consistent electric field. It is

$$
\begin{aligned}
P(\omega) & =-\frac{\omega}{2} \sum_{n, m, k}^{\epsilon_{n, m}^{k}>0}\left\{\phi_{e}(\omega)\right\}_{n, m}^{k} \operatorname{Im}\left[\frac{2 f_{n, m}^{k} \epsilon_{n, m}^{k}}{(\hbar \omega)^{2}-\left(\epsilon_{n, m}^{k}\right)^{2}}\left\{\phi_{s}(\omega)\right\}_{n, m}^{k}\right] \\
& =-\frac{\omega}{2} \sum_{n, m, k}^{\epsilon_{n, m}^{k}>0} \sqrt{2 f_{n, m}^{k} \epsilon_{n, m}^{k}}\left\{\phi_{e}(\omega)\right\}_{n, m}^{k} \operatorname{Im}\{\eta(\omega)\}_{n, m}^{k} .
\end{aligned}
$$

The Coulomb interaction may shift the poles of the matrix $\{\eta(\omega)\}_{n, m}^{k}$ from the Hartree single-particle excitations $\epsilon_{n, m}^{k}$ as can be seen from the eigenequation (7). The new poles will show up as resonances in the FIR absorption (10). Here a phenomenological broadening should be inserted to give the lifetime of the excitations. In a Fermi liquid the scattering cross-section decreases for lower energy one-particle excitations. Hence, one should expect that at least for low-energy one-particle excitations this broadening is small. The power absorption may be expanded in the eigenfunctions of the matrix $A$. The oscillator strengths can then be found and the whole FIR spectrum determined. Since the Hartree-RPA is a conserving approximation [12], the longitudinal f-sum rule for the oscillator strengths is satisfied for arbitrary electron-electron interaction strengths.

We study quantum wires with confining potentials of the form

$$
V_{c}(y)=\frac{1}{2} \hbar \omega_{0}\left[\left(\frac{y}{l_{0}}\right)^{2}+a\left(\frac{y}{l_{0}}\right)^{4}+b\left(\frac{y}{l_{0}}\right)^{6}\right],
$$

where $a$ and $b$ are parameters that determine the higher order deviations from parabolic confinement. By the method described above, we are able to find the FIR spectra arising from this confining potential.

\section{Numerical results}

The functional basis of the Hartree ground state has been chosen large enough so that a further expansion or iteration of the Hartree-equations does not result in visual changes to the single-particle energy spectra or the electron density $n_{s}(\vec{r})$ of the ground state. To attain sufficient accuracy in the calculation of the absorption the size of the functional basis for the excited states has been chosen such that further refinement results in changes to the location of the absorption peaks that are smaller than a typical linewidth in experiments $0.1 \mathrm{meV}$.

For the calculations we employ the usual GaAs parameters, $m^{*}=0.067 m_{0}, \kappa=12.4$, where $m_{0}$ is the the free electron mass. The absorption is only weakly dependent on $T$ in the temperature range $T \leq 4 \mathrm{~K}$ for the parameters we use. The calculations have thus been performed for $T=1.0 \mathrm{~K}$.

The FIR spectra have been calculated in the range of magnetic fields $B=0-3 \mathrm{~T}$. For a strict parabolic confinement, $a=0$ and $b=0$, we have checked that the generalised 
Kohns theorem is satisfied with a high degree of accuracy. We consider pure fourth order deviations, $a=0.01$ to $a=0.40$, and pure sixth order deviations, $b=0.001$ to $b=0.030$. Mixed deviations of fourth and sixth order do not give significant new qualitative information.

For small deviations, with either $a \neq 0$ or $b \neq 0$, a single anticrossing close to the $2 \omega_{c}$ line appears. The position of the anticrossing as a function of the deviation $a$ or $b$ is shown in Fig. 1. The horizontal line shows where $\Omega_{p}=2 \omega_{c}$, i.e. where a possible resonance between the magnetoplasmon and the cyclotron frequency should appear. The calculated anticrossing is to the right of the $2 \omega_{c}$-line for small deviations, but is shifted quite strongly to the left with increasing deviations. It is due to the interaction of the collective oscillations, the magnetoplasmons, with the first harmonic of the cyclotron resonance, $2 \omega_{c}$, a so called Bernstein mode[5].

The absorption peaks as a function of the magnetic field for increasing fourth order deviation, $a=0.03,0.10,0.20,0.40$, are shown in Fig. 2 and for increasing sixth order deviation, $b=0.003,0.005,0.010,0.030$, in Fig. 3. Only absorption peaks with an oscillator strength more than $1 \%$ of the total oscillator strength are shown. The absorption peaks are labeled strong, weak, very weak and extremely weak according to their relative strength for a given magnetic field. Only excitation energies in the range 3.5-8.5 meV are shown. The location of the strong resonance at zero magnetic field increases in energy with increasing deviation, because of the stronger confinement. However for intermediate magnetic fields the strong resonance develops a slightly negative slope with respect to the magnetic field. This softening of the mode increases with increasing deviations. For large deviations the simple splitting develops into a complex one, see Fig. \& for a fourth order deviation and Fig. 3 for a sixth order deviation. We see that strong deviations from a parabolic confinement leads to interactions with higher cyclotron harmonics, $n \omega_{c}$, where $n$ is larger than 2 . The new modes may also interact with each other. However it is not possible from the spectra to say which harmonics are involved, since several harmonics can be excited in a complicated way, and the anticrossing occurs at or in between the $n \omega_{c}$ lines. The spectrum is now very rich, because of the strong coupling of the collective motion to the internal motion in the wire. Splittings may also be seen on the weak resonance branches for $a=0.40$ in Fig. 2 and for $b=0.030$ in Fig. 3 .

The intensity as a function of the magnetic field and the supplied energy from the external potential is showed in Fig. \& for a pure fourth order deviation, $a=0.20$. Only resonances that have a strength of more than $1 \%$ are shown. We see that for this deviation there is mainly one anticrossing, all other resonances are weak. The softening of the modes occurs as long as two main resonances are seen, i.e as long as the modes are coupled.

The effects of the deviation is larger for a higher density of the electrons, since the 
chemical potential is higher and the 2DEG occupies states farther from the center of the wire that are more affected by the fourth and sixth order terms of the confinement.

\section{Summary}

The FIR spectrum for a wire with a confining potential deviating from a parabolic one was calculated in the time-dependent Hartree approximation. The anticrossing due to interaction of the magnetoplasmon with the first harmonic of the cyclotron resonance shifts strongly from the right of the $2 \omega_{c}$ intersection with the plasmon dispersion to the left with increasing deviations and the simple splitting develops into a more complex one, including interactions with higher harmonics of the cyclotron resonance.

In the quantum wire $\hbar \omega_{c}$ is not a characteristic single electron excitation energy, but the width of the system defines a characteristic wave vector which does not vanish with a decreasing deviation from the parabolic confinement. An external perturbation does excite plasma waves with a broad range of wave vectors, but the system responds in the strongest fashion for integer multiples of the 'natural wave vector'. In a homogeneous two-dimensional electron system the response is always with the same frequency and wave vector as the external perturbation, thus the asymptotic behaviour of the splitoff modes is simple. Here the asymptotic behaviour is complicated by the fact that the split-off modes again interact with higher order plasmons. A detailed study of this phenomena will be published elsewhere.

\section{Acknowledgments}

This research was supported in part by the Icelandic Natural Science Foundation, the University of Iceland Research Fund, and a NorFA Network Grant.

\section{References}

[1] Kohn W, Phys. Rev. 123, 1242 (1961).

[2] Pfannkuche D, Gerhardts R, Maksym P, and Gudmundsson V, Physica B 189, 6 (1993).

[3] Mal'shukov A G, Brataas A, and Chao K A, Phys. Rev. B 51, 7669 (1995).

[4] Gudmundsson V, Brataas A, Grambow P, Meurer B, Kurth T and Heitmann D, Phys. Rev. B 51, 17744 (1995).

[5] Bernstein I B, Phys. Rev. 109, 10 (1958).

[6] Patel C K N and Slusher R E, Phys. Rev. Lett. 21, 1563 (1968).

[7] Batke E, Heitmann D, Kotthaus J P, and Ploog K, Phys. Rev. Lett. 54, 2367 (1985).

[8] Batke E, Heitmann D, and Tu C W, Phys. Rev. B 34, 6951 (1986).

[9] Gerhardts R R and Gudmundsson V, Solid State Comm. 67, 845 (1988).

[10] Gudmundsson V, Gerhardts R R, Johnston R, and Schweitzer L, Z. Physik B 70, 453 (1988).

[11] Brataas A, Gudmundsson V, Mal'shukov A G, and Chao K A (unpublished). 
[12] Baym G and Kadanoff L P, Phys. Rev. 124, 287 (1961). 


\section{Figure captions}

Figure 1. The location of the first anticrossing as a function of the magnetic field $B$ and the fourth order deviation $a \neq 0, b=0(\diamond)$ or the sixth order deviation $a=0, b \neq 0$ $(+)$. The horizontal line shows where $\Omega_{p}=2 \omega_{c} .30$ states are occupied, $L_{x}=240 \mathrm{~nm}$, $\hbar \omega_{0}=3.94 \mathrm{meV}, T=1.0 \mathrm{~K}, m^{*}=0.067 m_{0}, \kappa=12.4$.

Figure 2. The location of the absorption peaks as a function of the magnetic field $B$ and the energy $E=\hbar \omega$ supplied by $\phi_{e}$ for a pure fourth order deviation. Only absorption peaks with an oscillator strength larger than $1 \%$ of the total oscillator strength are shown. The straight lines represent the harmonics of the cyclotron frequency, $n \times \omega_{c}(n=2,3,4,5)$. Strong absorption is marked by $\diamond$, weak one by + , very weak one by $\square$, and extremely weak one by $\times$. 30 states are occupied, $L_{x}=240 \mathrm{~nm}$, $\hbar \omega_{0}=3.94 \mathrm{meV}, T=1.0 \mathrm{~K}, m^{*}=0.067 m_{0}, \kappa=12.4$.

Figure 3. The location of the absorption peaks as a function of the magnetic field $B$ and the energy $E=\hbar \omega$ supplied by $\phi_{e}$ for a pure sixth order deviation. Only absorption peaks with an oscillator strength larger than $1 \%$ of the total oscillator strength are shown. The lines are the possible interaction of the magnetoplasmon with harmonics of the cyclotron frequency, $n \times \omega_{c}(n=2,3)$. Strong absorption is marked by $\diamond$, weak one by + , and very weak one by $\square$. 30 states are occupied, $L_{x}=240 \mathrm{~nm}$, $\hbar \omega_{0}=3.94 \mathrm{meV}, T=1.0 \mathrm{~K}, m^{*}=0.067 m_{0}, \kappa=12.4$.

Figure 4. The intensity of the absorption peaks as a function of the magnetic field $B$ and the energy $E=\hbar \omega$ supplied by $\phi_{e}$ for a pure fourth order deviation $a=0.20$. Only absorption peaks with an oscillator strength larger than $1 \%$ of the total oscillator strength are shown. 30 states are occupied, $L_{x}=240 \mathrm{~nm}, \hbar \omega_{0}=3.94 \mathrm{meV}, T=1.0 \mathrm{~K}$, $m^{*}=0.067 m_{0}, \kappa=12.4$. 


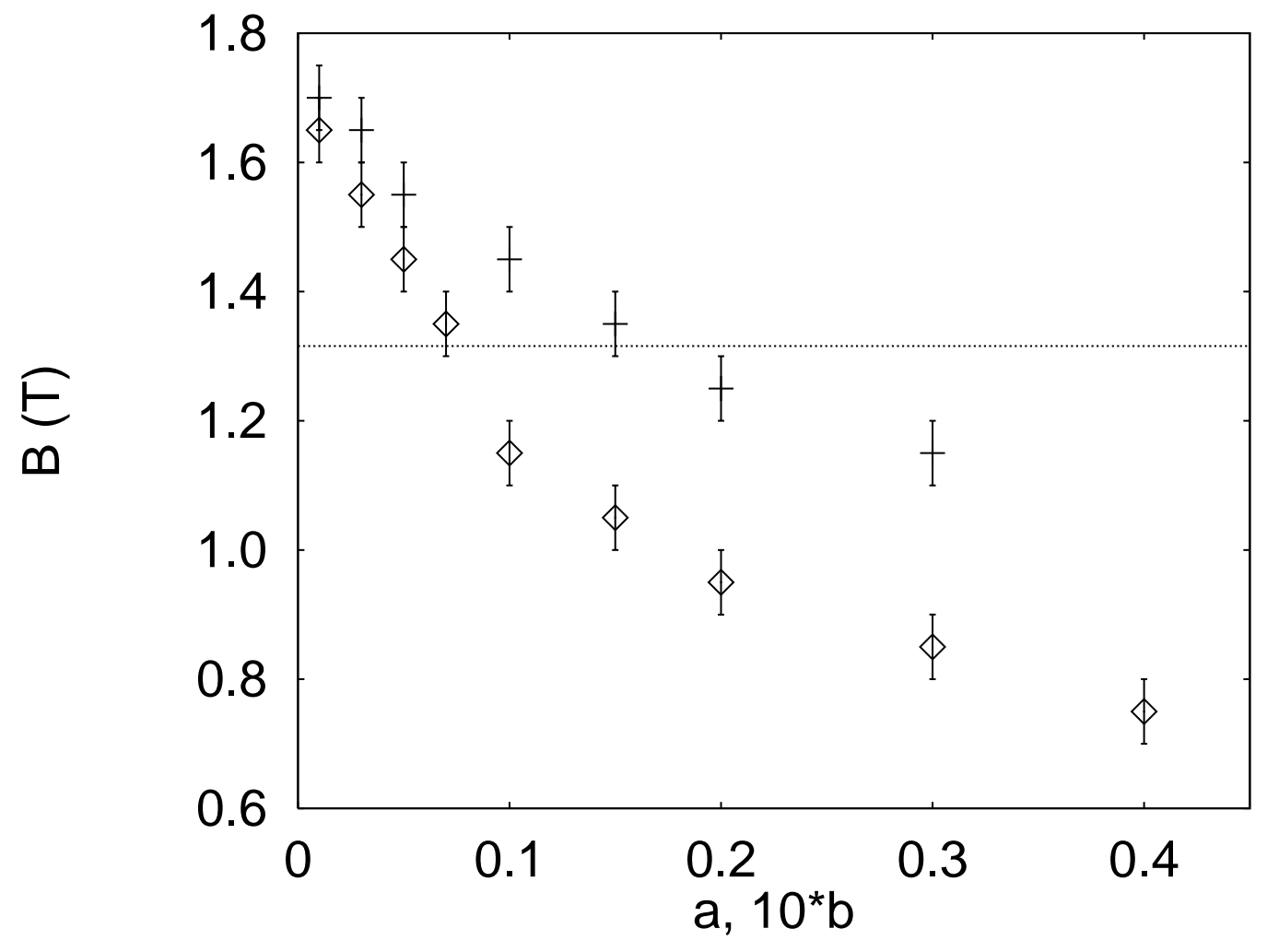

Brataas, Fig.1 


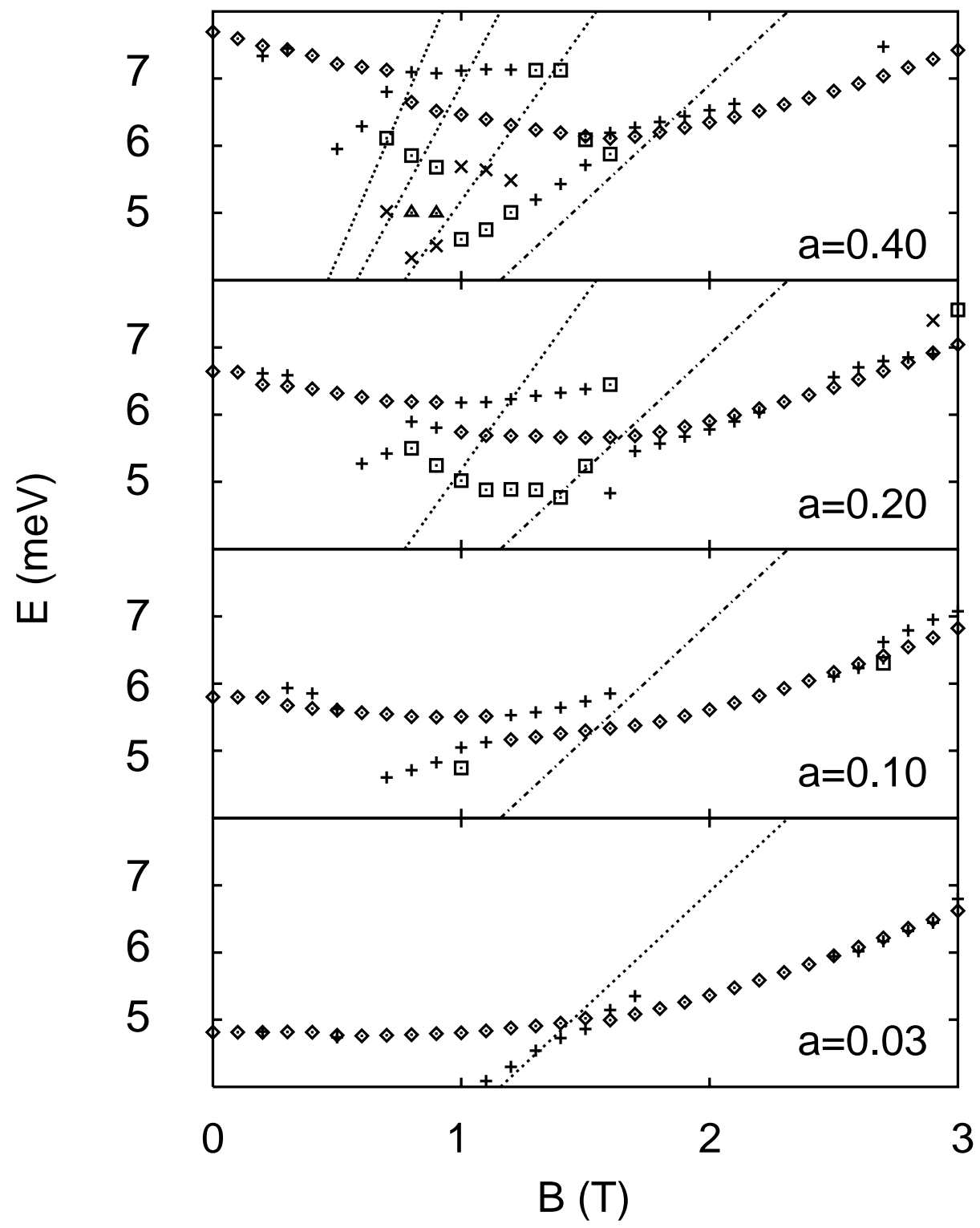

Brataas, Fig.2 


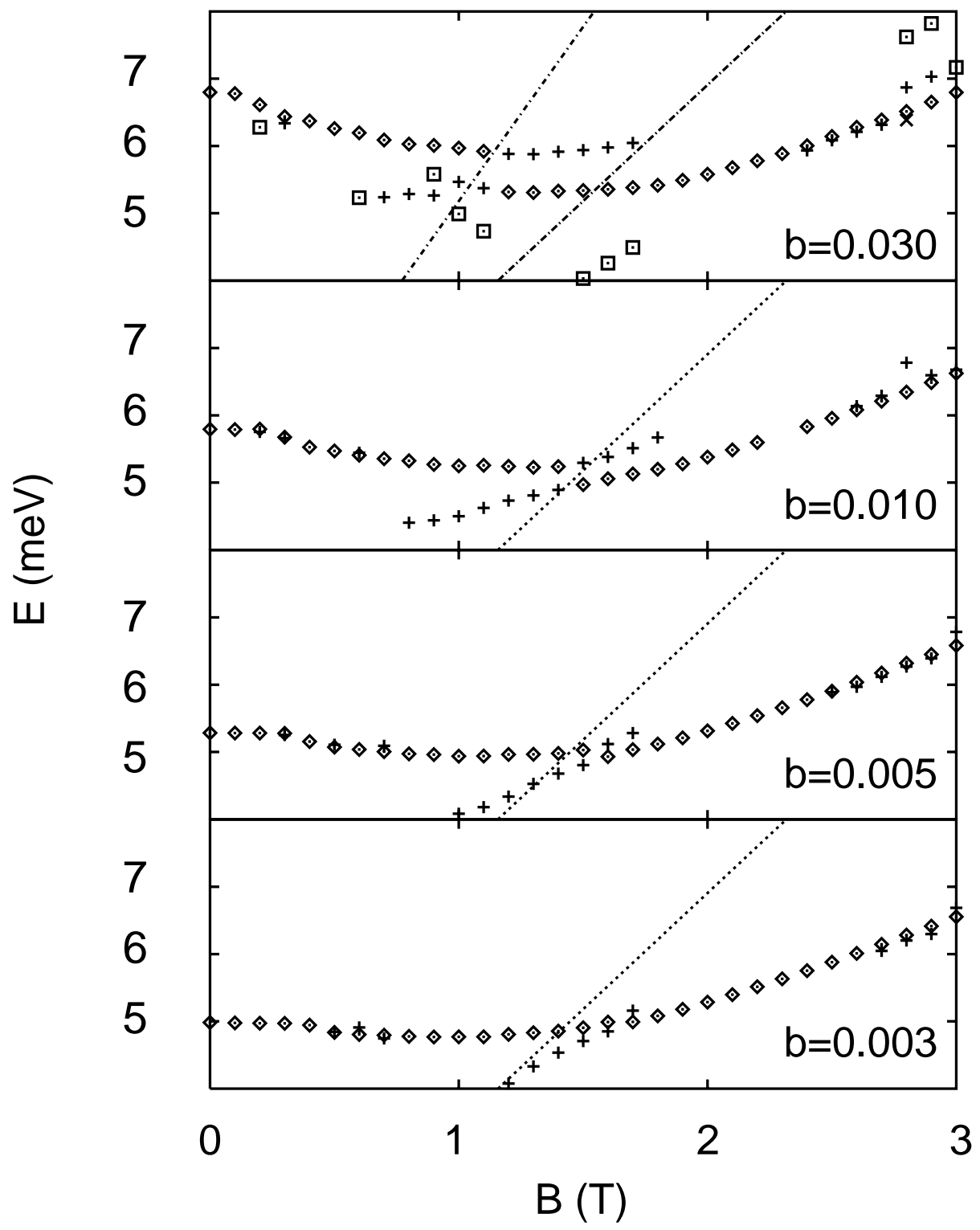

Brataas, Fig.3 


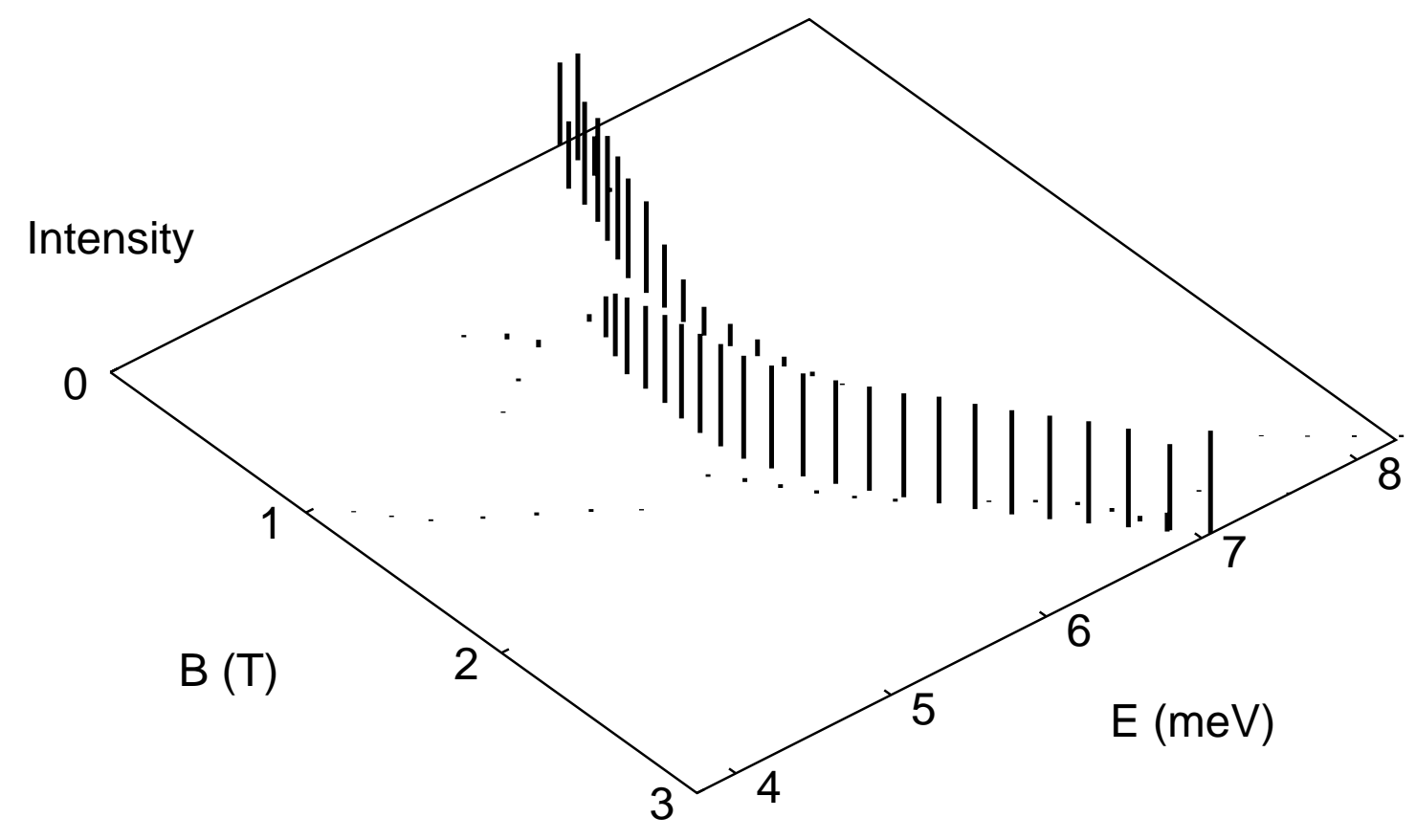

Brataas, Fig. 4 